\title{
Collagen XIX Alpha 1 Improves Prognosis in Amyotrophic Lateral Sclerosis
}

\begin{abstract}
Ana C. Calvo",*, Gabriela Atencia Cibreiro ${ }^{2}$, Paz Torre Merino², Juan F. Roy ${ }^{3}$, Adrián Galiana ${ }^{4}$, Alexandra Juárez Rufián², Juan M. Cano ${ }^{5}$, Miguel A. Martín ${ }^{6}$, Laura Moreno ${ }^{1}$, Pilar Larrodé1, Pilar Cordero Vázquez ${ }^{2}$, Lucía Galán ${ }^{7}$, Jesús Mora ${ }^{8}$, José L. Muñoz-Blanco ${ }^{9}$, María J. Muñoz ${ }^{1}$, Pilar Zaragoza $^{1}$, Elena Pegoraro ${ }^{10}$, Gianni Sorarù ${ }^{10}$, Marina Mora ${ }^{11}$, Christian Lunetta ${ }^{12}$, Silvana Penco $^{13}$, Claudia Tarlarini ${ }^{13}$, Jesús Esteban ${ }^{2}$, Rosario Osta ${ }^{1}$, Alberto García Redondo ${ }^{2}$

${ }^{1}$ LAGENBIO (Laboratory of Genetics and Biochemistry), Faculty of Veterinary-IIS, IA2-CITA, University of Zaragoza, Zaragoza, Spain. ${ }^{2}$ Neurology Department, ALS Unit, CIBERER U-723, Health Research Institute, October 12th Hospital "IIS I+12", Madrid, Spain. ${ }^{3}$ Ferkauf Graduate School of Psychology, Yeshiva University, NY 10461, USA. ${ }^{4}$ Servicio de Reumatología, Hospital Universitario de la Princesa, Instituto de Investigación Sanitaria La Princesa, Madrid, Spain. ${ }^{5}$ Orthopaedic Surgery Department, October 12th Hospital, Madrid, Spain. ${ }^{6}$ Grupo Enfermedades Mitocondriales y Neuromusculares, Instituto de Investigación Sanitaria Hospital 12 de Octubre (imas12), U723CIBERER, Madrid, España. ${ }^{7}$ Neurology Department, ALS Unit, Clínico Universitario San Carlos Hospital, Madrid, Spain. ${ }^{8}$ Neurology Department, ALS Unit, Carlos III Hospital, Madrid, Spain. ${ }^{9}$ Neurology Department, ALS Unit, Health Research Institute, Gregorio Marañón Hospital "IISGM", Madrid, Spain. ${ }^{10}$ Neurological Clinic, Department of Neurosciences, University of Padova, Padova, Italy. ${ }^{11}$ Muscle Cell Biology Laboratory, Neuromuscular Diseases and Neuroimmunology Unit, Fondazione IRCCS Istituto Neurologico C. Besta, Milan, Italy. ${ }^{12}$ NEMO (NEuroMuscular Omnicentre) Clinical Center, Fondazione Serena Onlus, Milan, Italy. ${ }^{13}$ Medical Genetics Unit, Department of Laboratory Medicine, Niguarda Ca' Granda Hospital, Milan, Italy.
\end{abstract}

[Received July 25, 2018; Revised September 15, 2018; Accepted September 17, 2018]

\begin{abstract}
The identification of more reliable diagnostic or prognostic biomarkers in age-related neurodegenerative diseases, such as Amyotrophic Lateral Sclerosis (ALS), is urgently needed. The objective in this study was to identify more reliable prognostic biomarkers of ALS mirroring neurodegeneration that could be of help in clinical trials. A total of 268 participants from three cohorts were included in this study. The muscle and blood cohorts were analyzed in two cross-sectional studies, while the serial blood cohort was analyzed in a longitudinal study at 6-monthly intervals. Fifteen target genes and fourteen proteins involved in muscle physiology and differentiation, metabolic processes and neuromuscular junction dismantlement were studied in the three cohorts. In the muscle biopsy cohort, the risk for a higher mortality in an ALS patient that showed high Collagen type XIX, alpha 1 (COL19A1) protein levels and a fast progression of the disease was $70.5 \%(P<0.05)$, while in the blood cohort, this risk was $20 \%(P<0.01)$. In the serial blood cohort, the linear mixed model analysis showed a significant association between increasing $C O L 19 A 1$ gene levels along disease progression and a faster progression during the follow-up period of 24 months $(P<0.05)$. Additionally, higher COL19A1 levels and a faster progression increased $17.9 \%$ the mortality risk $(P<0.01)$. We provide new evidence that COL19A1 can be considered a prognostic biomarker that could help the selection of homogeneous groups of patients for upcoming clinical trial and may be pointed out as a promising therapeutic target in ALS.
\end{abstract}

Key words: Collagen XIX type A1, ALS, prognostic biomarker, neurodegeneration, disease progression

Amyotrophic Lateral Sclerosis (ALS) is a devastating neurodegenerative disease that promotes a progressive motor neuron loss and muscle weakness. The survival period of time is within 2 to 10 years after the signs and

*Correspondence should be addressed to: Dr. Ana Cristina Calvo, Veterinary Faculty of Zaragoza, Health Research Institute of Aragon (IIS). University of Zaragoza, Miguel Servet, 177, 50013 Zaragoza, Spain. Email: accalvo@unizar.es.

Copyright: @ 2018 Calvo AC et al. This is an open-access article distributed under the terms of the Creative Commons Attribution License, which permits unrestricted use, distribution, and reproduction in any medium, provided the original author and source are credited. 
symptoms firstly appear [1,2]. A large proportion of people with ALS are sporadic cases and they lack of clear genetic association, while a small proportion of ALS patients, 5-10\%, correspond to familial ALS cases and they have a family history of ALS or a related condition called frontotemporal dementia (FTD). FTD is a progressive brain disorder that affects personality, behavior, and language and this disorder is present in $20 \%$ of the ALS patients [1]. The most known mutations that produce the typical adult-onset ALS phenotype in the familial cases are related to the copper/zinc superoxidedismutase-1 gene (SOD1), Tar DNA-binding protein gene $(T A R D B P)$ (previously known as TDP-43), DNA/RNAbinding protein called FUS (fused in sarcoma), TLS (translocation in liposarcoma), and the most recent hexanucleotide repeat expansion in C9ORF72 [1,3].

The complexity in the discovery of potential biomarkers for neurodegenerative diseases, especially in the case of ALS, relies greatly upon the lack of etiopathogenic origin in the whole population of ALS patients. In fact, ALS shares physiophatological abnormalities with other neuropathies such as Alzheimer's disease or Parkinson's disease and, more precisely, this close connection among these neurodegenerative diseases makes the identification of specific biomarkers for ALS a difficult task [4,5]. Dementia is also relatively frequent in ALS and may be a consequence of either frontotemporal lobar degeneration (FTLD) or a result of co-existing Alzheimer disease [6,7].

In this complex scenario and in spite of the numerous studies attempting to find specific gene/protein targets exclusive for ALS and characteristic of both familial and sporadic cases, the prognosis of the disease remains poor. Another main challenge relies on the fact that ALS is a multifactorial and relentlessly progressive disease, which hinders the use of an effective treatment. Therefore, the search for reliable biomarkers of the disease that can provide the identification of accurate indicators of early symptoms, disease progression, or even patients' survival, is urgently needed. It has been reported that older age at symptom onset, early respiratory muscle dysfunction, and bulbar-onset disease are associated with reduced survival, whereas limb-onset disease, younger age at presentation, and longer diagnostic delay are independent predictors of prolonged survival [8]. In this study, our main objective was to identify molecular biomarkers mirroring neurodegeneration in ALS patients that can also enable an earlier prognosis in the disease.

Previous studies by our research workgroup on transgenic SOD1G93A mice suggested five genes, Mef2c (myocyte enhancer factor 2C), Gsr (oxidative stress metabolism), Col19al (collagen, type XIX, alpha 1), Calm1 (calmodulin 1), and Snx10 (sorting nexin 10), as potential genetic biomarkers of longevity in transgenic
SOD1G93A mice, one of the best-characterized animal models for ALS that resembles both clinical and pathological characteristics of ALS patients $[9,10]$. Consequently, our next and main challenge was to analyze in depth the prognostic nature of the biomarkers identified in this animal model in ALS patients to correlate these biomarkers to the clinical variables routinely monitored in ALS patients to improve accuracy in the prediction. Mef $2 c$ gene is involved in muscle differentiation and regeneration [11], Gsr and Calml genes are involved in oxidative stress [12] and calcium homeostasis [13], respectively, Col19al gene is involved in the maintenance of muscle integrity [14] and SnxlO gene can play a relevant role in the muscle and bone dysregulation [15]. To achieve this challenge, we firstly aimed to test a panel of biomarkers in muscle biopsies from ALS patients and secondly, in blood samples to identify broadly applicable prognostic biomarkers for ALS disease progression in a non-invasive way. More in depth, the specific objective in this study was to look at the associations among molecular markers and clinical variables to identify more reliable prognostic biomarkers that could be of help in clinical trials. The findings in this study revealed that COL19A1 levels in muscle biopsies and blood samples from ALS patients can be considered a prognostic biomarker to accurately monitor the disease progression, especially in those patients that share a high disability in the first symptomatic stages of the disease.

\section{MATERIALS AND METHODS}

\section{Study approval}

Blood and muscle biopsy samples from patients were obtained with written informed consent prior to inclusion in the study to publication of their case details, which has been conducted according to Declaration of Helsinki principles, following the ethical rule of the October 12th Hospital (local Ethical Committee of Clinical Investigation approval reference 14/2007, Madrid, Spain), and according to the Directive 2004/23/EC of the European Parliament and of the Council. Participants were identified by number, not by name.

\section{Experimental Design}

This study included a total of 268 participants from three cohorts, matched for age and gender, whenever possible. For the muscle biopsy cohort, a total of 49 ALS patients, 24 healthy controls and 14 patients with other neuropathies (ONP) were included (Table 1). In the blood cohort, 59 ALS patients, 58 healthy controls and 24 ONP patients were included (Table 2). A second subgroup of forty sporadic ALS patients was monitored in the serial 
blood cohort at 6-monthly intervals during a follow-up period of 24 months from the symptom onset in each patient (Table 3). The muscle and blood cohorts were analyzed in two cross-sectional studies, while the serial blood cohort was analyzed in a longitudinal study. The participants were different in the three cohorts (Fig. 1).

Table 1. General and clinical characteristics of the study subjects in the muscle biopsy study cohort.

\begin{tabular}{|c|c|c|c|c|}
\hline \multirow{2}{*}{$\begin{array}{l}\text { Type of cohort }(\mathrm{N}) \\
\text { Patients' characteristics }\end{array}$} & & \multicolumn{3}{|c|}{ Muscle biopsy cohort* $(N=87)$} \\
\hline & & $\begin{array}{c}\text { ALS patients } \\
\quad(n=49)\end{array}$ & $\begin{array}{c}\text { ONP patients } \\
\quad(n=14)\end{array}$ & $\begin{array}{l}\text { Healthy controls } \\
\qquad(\mathrm{n}=24)\end{array}$ \\
\hline \multirow[t]{2}{*}{ Gender (n) } & Female & 15 & 8 & 19 \\
\hline & Male & 34 & 6 & 5 \\
\hline Age at illness onset (mean $\pm \mathrm{SD})$ & & $57.02 \pm 12.42$ & $43.79 \pm 16.02$ & \\
\hline Age at biopsy (mean $\pm \mathrm{SD})$ & & $58.65 \pm 12.52$ & $42.34 \pm 25.89$ & $55.07 \pm 26.07$ \\
\hline Disease duration, months (mean $\pm \mathrm{SD}$ ) & & $19.54 \pm 25.15$ & & \\
\hline \multirow[t]{9}{*}{ Clinical Phenotype (n) } & ALS, spinal & 42 & & \\
\hline & ALS, bulbar & 6 & & \\
\hline & ALS + FTD & 1 & & \\
\hline & OMD & & 5 & \\
\hline & SMA & & 5 & \\
\hline & CIDP & & 1 & \\
\hline & $\mathrm{KD}$ & & 1 & \\
\hline & NMD & & 1 & \\
\hline & $\mathrm{AD}$ (Early) & & 1 & \\
\hline ALSFRS-r at biopsy (mean \pm SD) & & $40.24 \pm 6.20$ & & \\
\hline \multirow[t]{5}{*}{ El Escorial criteria at onset (n) } & Unavailable & 33 & & \\
\hline & Defined & 1 & & \\
\hline & Probable & 6 & & \\
\hline & Possible & 5 & & \\
\hline & Suspected & 4 & & \\
\hline \multirow[t]{7}{*}{ Genetic Diagnosis (n) } & SALS & 26 & & \\
\hline & FALS & 6 & & \\
\hline & SALS + FTD & 2 & & \\
\hline & MND-FTD & 1 & & \\
\hline & PLS-SALS & 1 & & \\
\hline & PMA-SALS & 1 & & \\
\hline & Unavailable & 12 & & \\
\hline * All subjects were of caucasian ethic $\mathrm{g}$ & & & & \\
\hline
\end{tabular}

The clinical characteristics of the participants in each cohort were firstly assessed. In the ALS group, these characteristics included the revised ALS Functional Rating Scale-revised (ALSFRS-r), the ALSFRS-r slope
(48-ALSFRS-r /disease duration at time assessment), the clinical phenotype, the diagnostic delay, the age at onset, age at sampling, gender, the revised El Escorial criteria [16], the onset site and the genetic diagnosis. The 
ALSFRS-r provided an estimation of the patient's degree of functional impairment. In the serial blood cohort, ALSFRS-r was evaluated serially at 6-monthly intervals to objectively assess any progression of the disease at each sample extraction time. Additionally, the ALSFRS-r slope was calculated in both cross-sectional and longitudinal studies to monitor the rate of progression based on the functional scale of the patient. The classification system to estimate the ALSFRS-r slope was based on the previous system used by Elamin and coworkers [17]. The interpretation of this classification system is as follows: ALSFRS-R slope $<0.025$ points/month, 0.25-0.49 points/month, 0.50-0.99 points/month, and $\geq 1$ points/month. This means the higher ALSFRS-r slope $(\geq 1$ points/month), the faster functional decline.

Table 2. General and clinical characteristics of the study subjects in the blood study cohort.

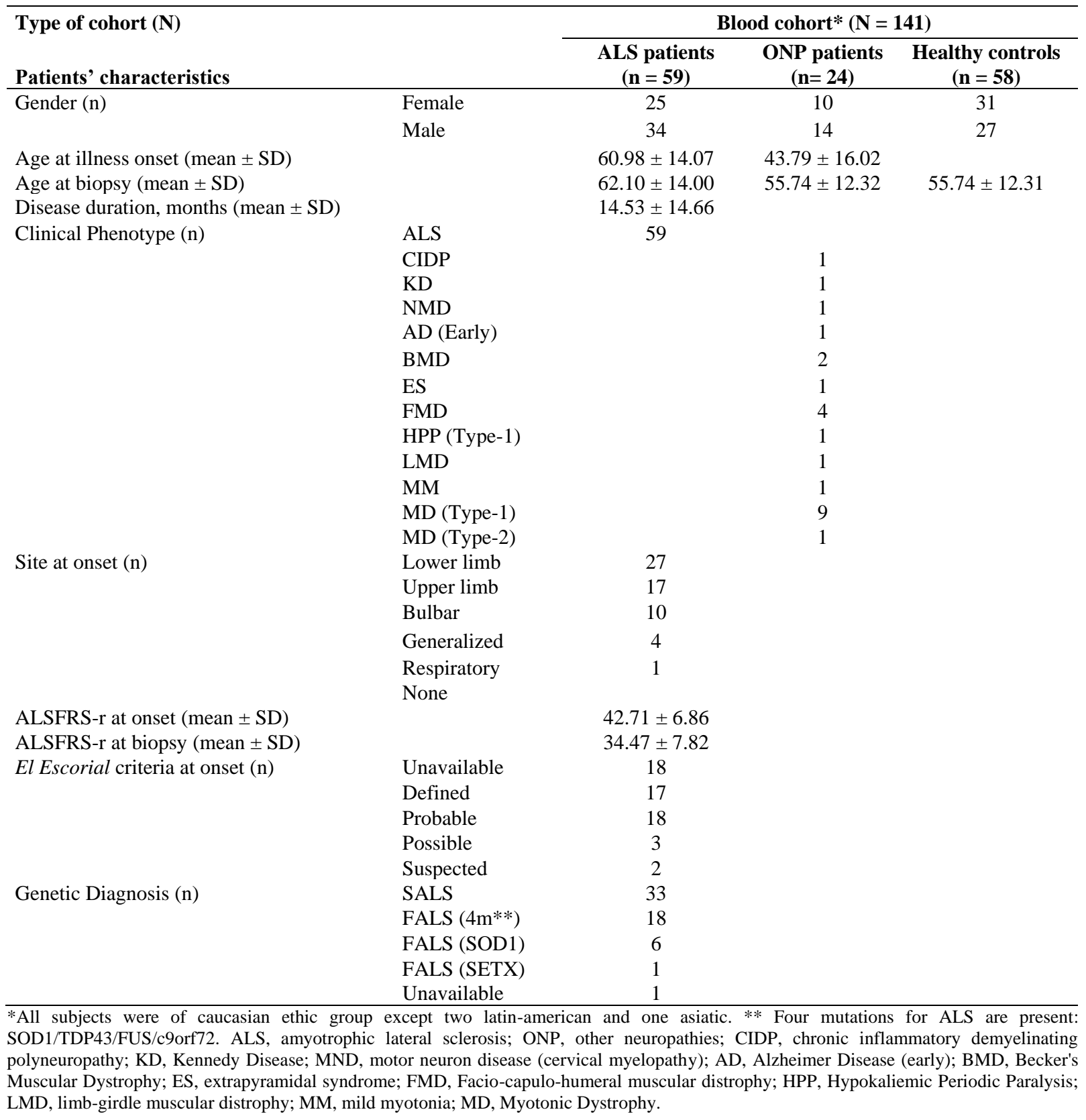




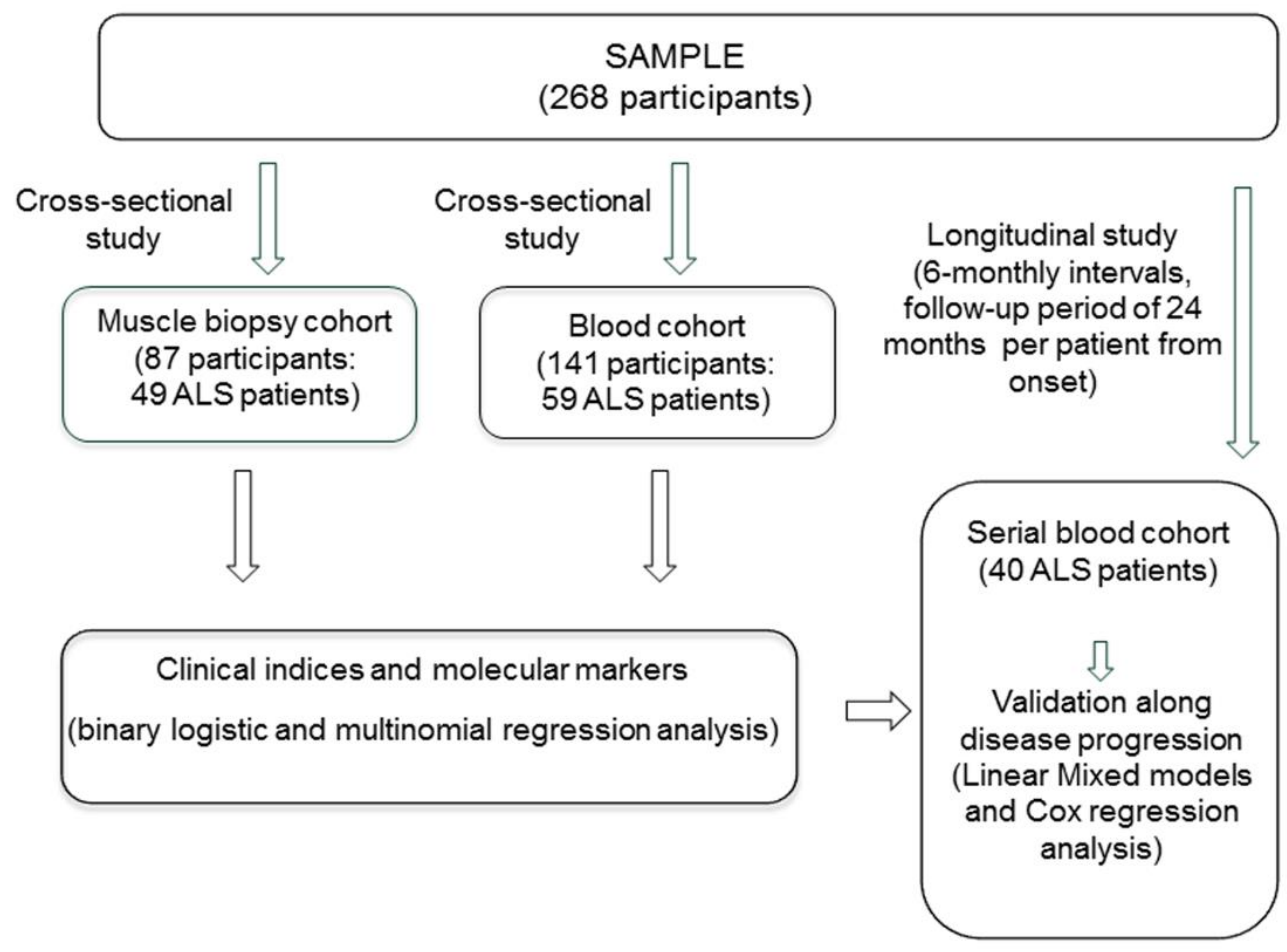

Figure 1. Flow diagram of the three study cohorts and overview of the study design. A total of 148 ALS patients were enrolled in two cross-sectional studies and one longitudinal study to detect associations among molecular markers and clinical variables in skeletal muscle biopsy (ALS muscle biopsy cohort) and blood samples (ALS blood cohort) and to identify prognostic biomarkers along the disease progression, especially in serial blood samples.

\section{RNA extraction and purification from muscle biopsies and blood samples}

Muscle biopsy samples were mainly obtained from brachial biceps using open biopsy after subcutaneous anesthesia administration and the samples were immediately frozen in liquid nitrogen. The biopsy sample from each individual was used to obtain RNA and protein extracts, using used the same methodology as the one employed in a previous study [18].

Blood samples were immediately processed and treated with a Ficoll gradient (Ficoll-PaqueTM Plus; GE Healthcare, Madrid, Spain). RNA extraction and purification were carried out following the manufacturer's instructions. In the case of blood samples received frozen in Pax tubes, the PAXgene Blood RNA Kit (PreAnalytiX, 8634 Hombrechtikon, Switzerland) was used to extract the RNA fraction. In all the muscle and blood samples, the cDNA was also obtained from $1 \mathrm{~g}$ of total extracted RNA (High Capacity cDNA RT kit; Applied Biosystems, Madrid, Spain). One muscle biopsy sample and one blood sample were obtained per participant from the muscle biopsy and blood cohorts, respectively. In the serial blood cohort, serial blood samples obtained every six months. In this case, a maximum of five serial samples were collected per patient, starting at the first age of sampling.

\section{Gene expression analysis}

The gene expression analysis was performed by real-time PCR (7500 Real-Time PCR System, Applied Biosystem, Madrid, Spain). Fifteen target genes were included in this analysis (Table 4). The Taqman probes (Applied Biosystems, Madrid, Spain) (Table 4) used in this study were tested and in all the cases the efficiency was near $100 \%$. All the real-time PCR reactions were done in triplicate and three endogenous genes (glyceraldehyde 3phosphate dehydrogenase, GAPDH, hypoxanthine phosphoribosyltransferase, HPRT, and TATA-binding protein, TBP) were used to normalize the target gene expression. Data was quantitatively analyzed with respect to a calibrator sample, selected from a control subject, using the $\Delta \Delta \mathrm{Ct}$ method. 
Table 3. General and clinical characteristics of sporadic ALS patients in the serial blood study cohort.

\begin{tabular}{|c|c|c|c|c|c|c|}
\hline \multicolumn{2}{|l|}{ Type of cohort $(\mathrm{N})$} & \multicolumn{5}{|c|}{ Serial blood cohort $*(N=40)$} \\
\hline & & \multicolumn{5}{|c|}{ ALS patients $(n=40)$} \\
\hline \multicolumn{2}{|l|}{ Patients' characteristics } & Onset & 6 months & 12 months & 18 months & 24 months \\
\hline \multirow[t]{2}{*}{ Gender (n) } & Female & 15 & & & & \\
\hline & Male & 25 & & & & \\
\hline Age at illness onset (mean \pm SD) & & $57.02 \pm 12.42$ & & & & \\
\hline $\begin{array}{l}\text { Disease duration, months (mean } \pm \\
\text { SD) }\end{array}$ & & $57.53 \pm 23.48$ & & & & \\
\hline \multirow[t]{3}{*}{ Clinical Phenotype (n) } & $\begin{array}{l}\text { SALS, UMN and } \\
\text { LMN spinal }\end{array}$ & 25 & & & & \\
\hline & SALS, UMN spinal & 1 & & & & \\
\hline & SALS, LMN spinal & 14 & & & & \\
\hline ALSFRS-r $($ mean \pm SD) & & $27.8 \pm 5.47$ & $23.8 \pm 5.94$ & $19.6 \pm 6.27$ & $18.5 \pm 6.76$ & $17.6 \pm 6.76$ \\
\hline \multirow[t]{3}{*}{ El Escorial criteria at onset (n) } & Defined & 25 & & & & \\
\hline & Possible & 4 & & & & \\
\hline & Probable & 11 & & & & \\
\hline Forced vital capacity (FVC) (\%) & & $10.4 \pm 6.11$ & $14.6 \pm 6.99$ & $15.0 \pm 10.44$ & $17.8 \pm 11.12$ & $20.6 \pm 15.74$ \\
\hline Body mass index (BMI) $\left(\mathrm{Kg} / \mathrm{m}^{2}\right)$ & & $26.5 \pm 3.81$ & $26.3 \pm 3.82$ & $25.9 \pm 4.11$ & $26.0 \pm 3.71$ & $25.3 \pm 3.55$ \\
\hline Genetic Diagnosis (n) & SALS & 40 & & & & \\
\hline
\end{tabular}

\section{Protein expression analysis}

Fourteen target proteins were included in this analysis (Table 5). We used the same methodology as the one employed in a previous study [18]. In this study, the protein extracts were subjected to $10 \%$ SDSpolyacrylamide gel electrophoresis. The corresponding primary antibody was diluted 1:500 in blocking buffer and glyceraldehyde-3-phosphate dehydrogenase (GAPDH, Santa Cruz Biotechnology, Quimigen S.L., Madrid, Spain) was used as housekeeping protein to normalize protein levels in target proteins. The integrated optical density (IOD) values of the target proteins were accurately normalized with respect to the housekeeping protein IOD values.

Table 4. Taqman ${ }^{\circledR}$ probe and primer mixtures used in gene expression assays.

\begin{tabular}{lll}
\hline \multicolumn{1}{c}{ Gene name } & \multicolumn{1}{c}{ Gene Symbol } & \multicolumn{1}{c}{ Assay ID } \\
\hline Ankyrin repeat domain 1 (cardiac muscle) & ANKRD1 & Hs00173317_m1 \\
Collagen, type XIX, alpha 1 & COL19A1 & Hs00156940_m1 \\
F-box protein 32 & FBXO32 & Hs01041408_m1 \\
Glycogen synthase kinase 3 & GSK3 & Hs01047719_m1 \\
Glutathione reductase & GSR & Hs00167317_m1 \\
Inositol(myo)-1(or 4)-monophosphatase 1 & $I M P A 1$ & Hs04188597_m1 \\
Myocyte enhancer factor 2C & MEF2C & Hs00231149_m1 \\
Myogenin (myogenic factor 4) & MYOG & Hs01072232_m1 \\
Reticulon 4 & RTN4 (NOGO A) & Hs00199671_m1 \\
Ras-related associated with diabetes & RRAD & Hs00188163_m1 \\
Senataxin & SETX & Hs00209294_m1 \\
Sarcolipin & SLN & Hs01888464_s1 \\
Sorting nexin 10 & SNX10 & Hs00203362_m1 \\
Superoxide dismutase 1, soluble & SOD1 & Hs00533490_m1 \\
Vacuolar protein sorting 54 homolog (S. cerevisiae) & VPS54 & Hs00212957_m1 \\
\hline
\end{tabular}


Table 5. Primary antibodies used in protein expression assays.

\begin{tabular}{lll}
\hline Protein name & Protein Symbol & Reference number, primary antibodies \\
\hline Anti-Apoptosis-inducing factor, mitochondrion-associated, 1 & AIFM1 & SAB2100079, anti-rabbit, Sigma-Aldrich \\
Ankyrin repeat domain 1 (cardiac muscle) & ANKRD1 & SAB1101413, anti-rabbit antibody, Sigma-Aldrich \\
Aspartate transcarbamylase & CAD & SAB2100334, anti-rabbit, Sigma-Aldrich \\
Collagen, type XIX, alpha 1 & COL19A1 & H00001310-A01, anti-mouse, Novus Biologicals \\
Coenzyme Q3 homolog & CoQ3 & WH0051805M1, anti-mouse, Sigma-Aldrich \\
F-box protein 32 & FBXO32 & ab67866, anti-mouse, ABCAM \\
Glutathione reductase & GSR & WH0002936M1, anti-mouse, Sigma-Aldrich \\
Inositol(myo)-1(or 4)-monophosphatase 1 & IMPA1 & SAB2103588, anti-rabbit, Sigma-Aldrich \\
Myogenin (myogenic factor 4) & MYOG & WH0004656M1, anti-mouse, Sigma-Aldrich \\
Anti-CoQ1 & PDSS1 & AV46195, anti-rabbit, Sigma-Aldrich \\
Reticulon 4 & RTN4 (NOGO A) & R3282, anti-rabbit, Sigma-Aldrich \\
Sarcolipin & SLN & ab25860, anti-rabbit, ABCAMK \\
Sorting nexin 10 & SNX10 & WH0029887M1, anti-mouse, Sigma-Aldrich \\
Vacuolar protein sorting 54 homolog (S. cerevisiae) & VPS54 & SAB1401701, anti-mouse, Sigma-Aldrich \\
\hline
\end{tabular}

\section{Statistical analysis}

The normality of data was verified by means of the Kolmogorov-Smirnov test. The data that did not show a homogeneous distribution was analyzed using KruskalWallis tests to compare means, while the homogeneous distribution of the data was studied using Student's-t test. All values were expressed as the mean \pm standard error of the mean (SEM). The area under ROC curves (AUC) was calculated, following the same methodology used in a previous study to explore the support for diagnosis nature of the molecular markers under study [19]. Binary and multinomial logistic regression analysis was used to investigate the influence of molecular markers on the clinical variables. All the clinical variables were converted to categorical variables in the whole regression analysis. Cox proportional hazards regression analysis was undertaken in case of continuous variables, such as COL19A1 levels, to evaluate their prognostic nature. Patients who were alive at the time of analysis were censored. After identifying significant predictors of prognosis on multivariate analysis, internal validation of the model was carried out using boot-strapping techniques using 1000 random samples to obtain $95 \%$ confidence. Leave-one-out cross validation was used to confirm the accuracy in each regression analysis. Linear mixedeffects models were used to estimate the influence of COL19A1 levels on outcomes in the longitudinal study. The fixed effect of the baseline value of the outcome and the period of time in months on outcomes was modeled. Random intercepts were used to model between-patients variability. Statistical analysis was performed using SPSS Statistics version 22 (IBM, Spain). A two-sided significance level of 5\% was considered statistically significant.

\section{RESULTS}

\section{COL19A1 levels were strongest predictors of survival in the muscle biopsy cohort}

The expression profile of fifteen genes and fourteen proteins was analyzed in the muscle biopsy cohort and compared to healthy subjects and to ONP groups. Only COL19A1 gene and protein levels were significantly different in the ALS patient group with respect to healthy controls and the ONP groups. Remarkably, COL19A1 protein levels were approximately five-fold increase in the ALS patient group respect to healthy controls and the ONP groups in, while COL19A1 gene levels were approximately thirty-fold increase in ALS patients respect to the control groups (Fig. 2A). Furthermore, comparison of COL19A1 expression profile with the Area under ROC curves (AUC) was performed to test if COL19A1 levels could have a good performance as support for diagnosis tool. In order to assess predictive values, selected cut-off values were set for COL19A1 gene (COL19A1 gene >7), and for COL19A1 protein (COL19A1 protein $>1$ ) to minimize false positive cases (Fig. 2B). The parallel combination of data improved performance related to predictive values, yielding a $71.0 \%$ sensitivity and $100 \%$ specificity with a positive predictive value (PPV) of $99.9 \%$ and a negative predictive value (NPV) of 99.9\% (likelihood ratio, LR: 6.00, confidence interval, CI: 2.471-14.571).

We next explored the prognostic nature of COL19A1 gene in relation to the clinical parameters measured in ALS patients. The ALSFRS-r score provides an estimation of the patient's degree of functional impairment. Since the 1990s, this functional rating scale has been developed to measure the disease progression in 
the patients from time at onset to time at diagnosis and follow-up visits in the clinic. This scale is based on a set of twelve questions graded on a scale of 0 to 4 . The responses obtained in this questionnaire can shed light on the different domains that might be affected by the disease. The new and revised scale considers 48 points as the maximum score for each question [20]. It has been described that the rate of deterioration in the patient correlates with survival and, at the same time, it also correlates with a lower score in the ALSFRS-r scale, suggesting approximately an average loss of a point per month along the disease progression and therefore a higher disability in the patient [20]. Spearman correlation between ALSFRS-r score at sampling and COL19A1 gene levels was found significant $(\mathrm{r}=-0.349, P=0.025)$, which suggested that high COL19A1 levels could be related to lower ALSFRS-r scores at sampling and a higher disability in the patient. Binary logistic regression analysis showed that ALS patients that exhibited
ALSFRS-r scores at sampling higher or equal than 40, also showed a probability of expressing lower COL19A1 gene and protein levels than ALS patients with ALSFRSr scores at sampling lower than 40 (COL19A1 gene levels: odds ratio (OR): $0.839,95 \%$ confidence of interval $(\mathrm{CI})$ : 0.714-0.987, $P=0.034$; COL19A1 protein levels: OR: 0.977, CI: 0.877-1.089, $P=0.674)$. In addition, Spearman correlation between disease duration and diagnostic delay (time from symptom onset to diagnosis) was found significant $(\mathrm{r}=0.579, P<0.0001)$ as well as between disease duration and ALSFRS-r slope at sampling ( $r=$ $0.761, P<0.0001)$ and between diagnostic delay and ALSFRS-r slope at sampling $(\mathrm{r}=-0.556, P<0.0001)$. In this sense, ALS patients that showed a longer diagnostic delay (more than one year) also showed longer disease duration and a slow progression of the disease (lower ALSFRS-r slope scores, lower than 1 points/month).
A

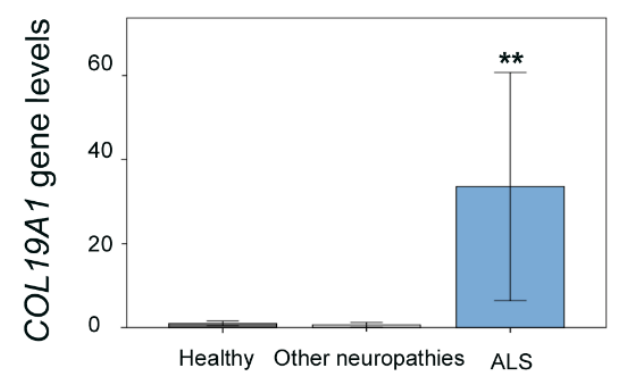

Simplified clinic presentation
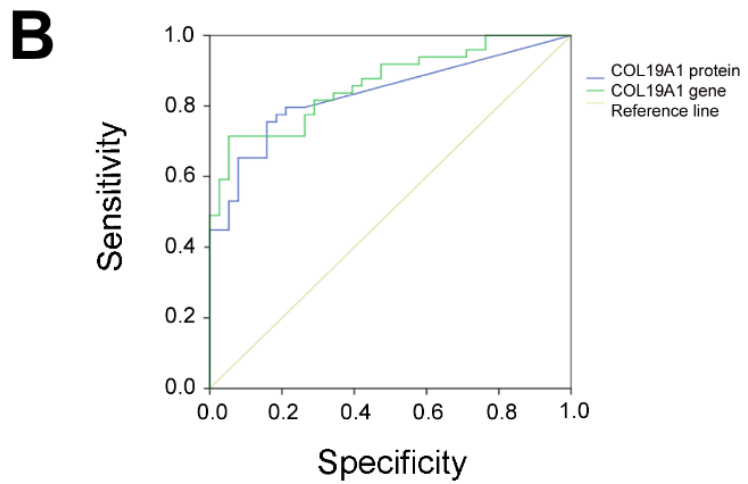

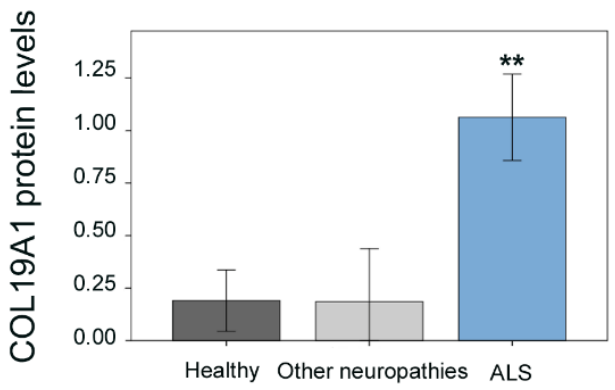

Simplified clinic presentation

\begin{tabular}{|l|c|c|c|}
\hline \multicolumn{1}{|c|}{ Biomarker } & AUC & SE & p-value \\
\hline COL19A1 protein & 0.833 & 0.044 & 0.000 \\
COL19A1 gene & 0.863 & 0.038 & 0.000 \\
\hline
\end{tabular}

Figure 2. Relative gene and protein expression levels of COL19A1 in the muscle biopsy study cohort. Gene and protein expression levels of COL19A1 in healthy subjects, ALS patients and other neuropathies patients (A). Kruskal Wallis tests showed significant differences among ALS patients and the other two groups under study, when gene and protein expression levels were tested $(\mathrm{P}<0.001, * *)$. (B) Areas under ROC curves (AUC) of gene and protein expression of COL19A1 were calculated to test its support for diagnosis criterion in ALS patients $(\mathrm{P}<0.001, * *)$. A total of 49 ALS (FALS and SALS) participants were included in this study and matched with 24 control individuals, and 14 ONP; SE: standard error. 
A

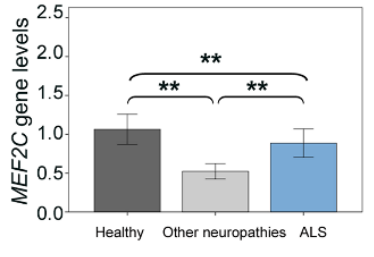

Simplified clinic presentation

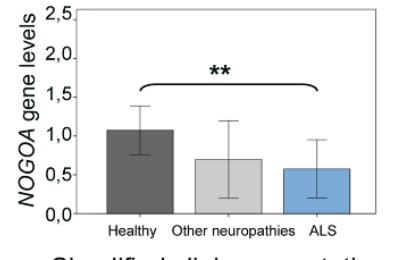

Simplified clinic presentation

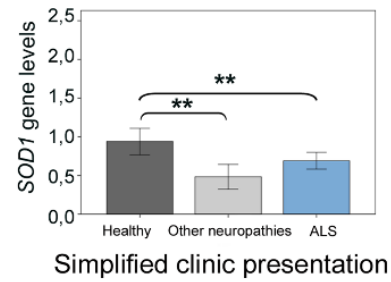

Simplified clinic presentation

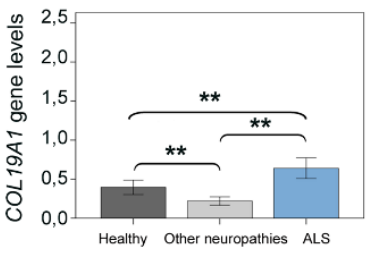

Simplified clinic presentation

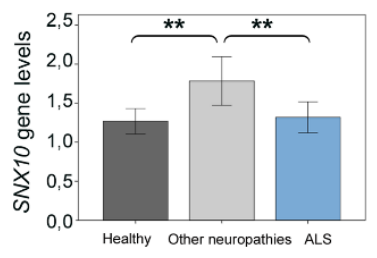

Simplified clinic presentation

B
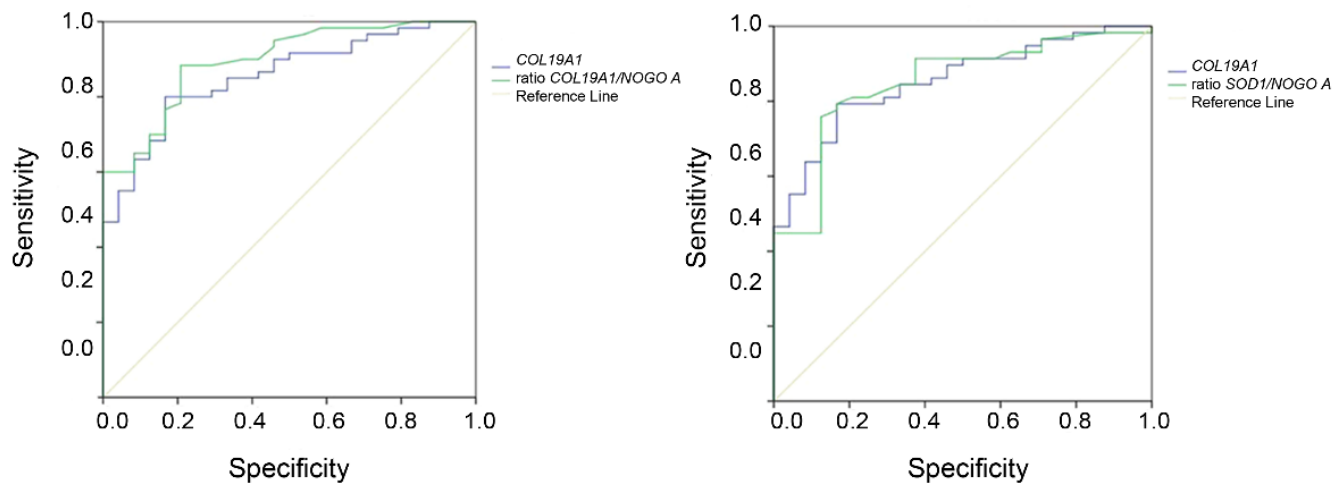

Figure 3. Relative gene expression analysis in the blood study cohort. Transcriptional expression levels of $M E F 2 C$, NOGO A, SOD1, COL19A1 and SNX10 in healthy subjects, ALS patients and other neuropathies patients. (A) Kruskal Wallis tests showed significant differences among ALS patients and healthy participants in all the cases except for SNX10. Significant differences among other neuropathies patient group and ALS patient group were found in all the cases except for NOGO A and SOD1. (B) Area under ROC curves of COL19A1 and the ratios COL19A1/NOGO A and SOD1/NOGO A were calculated in the sporadic ALS patient group. Significant differences were found between COL19A1 and the ratio COL19A1/NOGO A, and between COL19A1 and the ratio SOD1/NOGOA. A total of 141 participants were included in this study: 58 control individuals, 24 other neuropathology's individuals and 59 ALS patients (FALS and SALS patients); (*P $<0.05 ; * * \mathrm{P}<0.001)$, SE: standard error.

The next step was to investigate the role of COL19A1 levels in the disease progression, the diagnostic delay and the ALSFRS-r slope at sampling. Linear regression analysis suggested the disease duration correlated positively with the diagnostic delay (B coefficient: 0.820, CI: $0.452-1.189, P=0.008)$ and negatively with the ALSFRS-r slope (B coefficient: -10.587, CI: -19.586-(1.588), $P=0.023)$ and with low COL19A1 protein levels (B coefficient: $-14.407, \mathrm{CI}$ : $-30.228-1.414, P=0.043$ ), suggesting the higher ALSFRS-r slope scores (faster progression of the disease) and the higher COL19A1 protein levels, the shorter disease duration. Additionally, the diagnostic delay correlated positively with the disease duration in ALS patients that exhibited low COL19A1 gene levels (B coefficient: 0.909, CI: 0.556-1.263, $P<0.0001$ ), suggesting that low COL19A1 gene levels were closely associated with ALS patients that showed a longer disease duration and therefore, a diagnostic delay higher than one year. 
Finally, to validate the prognostic nature of COL19A1 expression levels multivariable Cox proportional hazards regression model was performed using leave-one-out cross validation. This analysis suggested that the lifespan in ALS patients showing COL19A1 protein levels above mean average (1.06) and additionally a fast progression of the disease (ALSFRS-r slope scores at sampling $\geq 1$ points/month) could be reduced $70.5 \%$ (ALSFRS-r slope, hazard ratio, HR:
1.569, CI: $1.245-1.976, P=0.000 ;$ COL19A1 protein levels, HR: 2.158, CI: 1.020-4.566, $P=0.044$ ), emphasizing the significance of COL19A1 levels as a useful, feasible, and potentially prognostic factor in patients with ALS. Since COL19A1 levels were found strongest predictors of survival in the ALS muscle biopsy cohort, we next investigated a second cohort of patients, the blood cohort, to validate these findings.

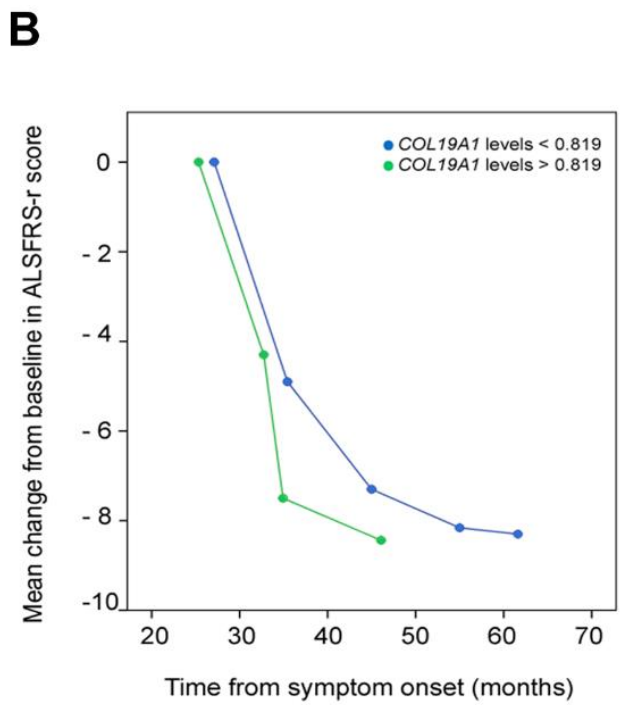

Figure 4. Linear mixed model analysis in the serial blood study cohort. (A) Linear mixed model analysis showed a significant relationship among a faster disease progression and high COL19Al levels from symptom onset, each dashed line corresponds to the COL19A1 levels from each patient. (B) Mean change from baseline in the ALSFRS-r score in patients that were monitored at 6-monthly intervals during a follow-up period of 24 months from the symptom onset that show COL19A1 levels above (green dot and line) and below (blue dot and line) average at first evaluation. Student's-t test was performed to analyze statistical differences between groups $(\mathrm{P}<0.001)$. A total of 40 SALS patients from the serial blood study cohort were included.

\section{The prognostic nature of COL19A1 levels was also validated in blood samples from ALS patients}

The gene expression profile of fifteen genes was analyzed in the ALS blood cohort and compared to the healthy group of subjects and ONP patients. Results showed that only COL19A1 and MEF2C levels were significantly deregulated in the ALS patient group compared to healthy controls and the ONP groups (Fig. 3A). Albeit COL19A1 levels were found significantly higher in the ALS patient group respect to the healthy and ONP groups, this difference in the gene expression levels was not so remarkable than the difference observed in the muscle biopsy cohort (approximately 1.75-fold change respect to the healthy control group and 2.35 -fold change respect to the ONP group), which is in accordance with the fact that COL19A1 gene is mostly expressed and related to the skeletal muscle tissue, rather than blood tissue. ROC curves analysis showed that the sensitivity and specificity ratios between COL19A1 and COL19A1/NOGO A and SOD1/NOGOA ratios were found statistically significant in the sporadic ALS patients' group (Fig. 3B). Selected cut-off values were set for COL19A1 gene (COL19A1 gene >0.847), and for COL19A1/NOGO A ratio (ratio COL19A1/NOGO A >0.650), as well as for SOD1/NOGO $A$ (ratio SODI/NOGO $A>0.960$ ) to minimize false positive cases. These results showed that a parallel combination of COL19A1 and the ratio COL19A1/NOGO $A$ improved performance, yielding a $78.7 \%$ sensitivity, while in the case of COL19A1 and the ratio SOD1/NOGO $A$, the sensitivity reached $70.7 \%$ (LR 6.214, CI 2.44915.771). Consequently, the relationship between COL19A1 levels and the ratios COL19A1/NOGO A and SODI/NOGO A could be more indicative of muscle damage in the population of sporadic ALS patients. 
Our next step was to investigate the relationship of these genes with the functional state in ALS patients. Spearman correlations between ALSFRS-r score at onset and COL19A1 levels ( $\mathrm{r}=-0.345, P=0.001), M E F 2 C(\mathrm{r}=$ $0.228, P=0.038)$ and $S N X 10(\mathrm{r}=0.494, P=0.000)$ gene levels were found significant, which suggested that high COL19A1 levels could be related to lower ALSFRS-r scores, indicating a high disability in the patient, while high $M E F 2 C$ and $S N X 10$ levels were more associated with a lower disability in the patient. Furthermore, COL19A1 levels correlated negatively with the diagnostic delay $(\mathrm{r}=$ -0.210, $P=0.040$ ), reinforcing that COL19A1 levels could be involved in a faster progression of the disease.

To investigate more in depth these correlations, binary logistic regression analysis was performed. We next explored the possible associations of these genes and the clinical variables measured in each patient that included ALSFRS-r at onset and at sampling, the diagnostic delay, age at onset, the revised El Escorial criteria, the onset site and the genetic diagnosis.

COL19A1 levels were associated with an age at onset lower than 55 years (OR: 2.967, CI: 1.213-7.257, $P=0.017$ ), a defined El Escorial criteria (OR: 3.050, CI: 1.266-7.350, $P=0.013$ ) and ALSFRS-r scores equal or lower than 40 (OR: 2.433, CI: 0.970-6.105, $P=0.058$ ), which is in accordance with the results observed in the muscle biopsy cohort. Therefore, high COL19A1 levels in blood could be also indicative of a worsening of the functional state and a high disability in the patient. Additionally, COL19A1 levels were also associated with a diagnostic delay lower than one year (OR: 0.953, CI: 0.909-0.998, P=0.043). In contrast, SNX10 and NOGO A levels were more associated with ALSFRS-r scores at onset higher than 40 (SNX10, OR: 0.381, CI: 0.213-0.679, $P=0.001$; NOGO A, OR: 0.366, CI: 0.184-0.725, $P=0.004)$, and an age at onset higher than 55 years (OR: 0.333, CI: $0.167-0.665, P=0.002$ ), suggesting that high SNX10 and NOGO A levels could be related to a lower disability in the patient. Interestingly, only in the case of $M E F 2 C$ levels, a significant association was found between high levels of this gene and the bulbar and upper limb onset site of the disease (OR: 2.963, CI: 1.171-7.497, $P=0.022$ ), which could indicate an advanced muscle damage. In addition, COL19A1 levels were associated with the sporadic form of the disease (OR: 13.771, CI: 2.520-75.255, $P=0.002$ ), while $S N X 10$ levels were more associated with the familial form of the disease (OR: 0.454, CI: 0.268-0.767, $P=0.003$ ).

Our next step was to unravel which of these genes could influence the clinical features in the patients and therefore, multivariate logistic regressions were performed. SNX10 levels were found significantly associated with the familial ALS patients' group (OR: 0.489 , CI: $0.268-0.892, P=0.020$ ), with ALS patients with an age at onset higher than 55 years (OR: 0.351, CI: $0.173-0.714, P=0.004$ ), and with patients showing a possible, probable or suspected El Escorial criteria at onset (OR: 0.417, CI: 0.211-0.822, $P=0.012$ ). These findings suggested that SNX10 levels could be indicative of a late worsening in the functional state of the ALS patients. In contrast, COL19A1 levels were found significantly associated with sporadic ALS patients (OR: 16.106, CI: 2.294-113.053, $P=0.005)$, with patients that share an age at onset lower than 55 years (OR: 2.543, CI: 1.007-6.422, $P=0.048)$, and a defined El Escorial criteria (OR: 2.955, CI: 1.214-7.192, $P=0.017$ ), highlighting that COL19A1 levels could be more indicative of an earlier worsening in the functional state in the ALS patients.

Finally and after analyzing the prognostic nature of the genes along the disease progression, Cox proportional hazards regression model suggested that COL19A1 gene levels above average (0.819) and a fast progression of the disease, based on high scores of the ALSFRS-r slope at onset ( $\geq 1$ points/month) could favored a shorter lifespan in ALS patients (ALSFRS-r slope, HR: 5.798, CI: 3.4059.872, $P<0.001$; COL19A1 levels, HR: 2.291, CI: 1.4355.945, $P=0.003)$. Notwithstanding, ALSFRS-r slope at sampling was also considering to be a potential predictor of disease progression, improving the accuracy of the prognostic prediction. Cox regression analysis, using leave-one-out cross validation, suggested that COL19A1 gene levels above mean average (0.819) and high ALSFRS-r slope at sampling scores above mean average ( $\geq 1$ points/month) could promote a $20 \%$ reduction in the lifespan of ALS patients (COL19A1, HR: 2.114, CI: 1.678-2.772, $P=0.019$, ALSFRS-r slope at sampling, HR: 2.368, CI: 1.763-3.181, $P<0.001)$.

These findings reinforced the potential role of COL19A1 levels as strongest predictors of disease progression in this cross-sectional study, in which only one sample of blood was extracted from each participant and analyzed. The validation of these findings in serial blood samples obtained in a longitudinal study could finally unravel the prognostic nature of COL19A1 levels in ALS patients, and especially in sporadic cases to which COL19A1 was significantly associated.

\section{High COL19A1 levels enabled a more precise prognosis in patients that shared a high disability in the first symptomatic stages}

Blood serial samples obtained from the same patient can provide useful information about the variation of the expression profile of any molecular marker along the time. In addition, they can be easily monitored and studied in parallel with the clinical variables that each patient exhibit during the progression of the disease, especially the ASFRS-r scores and ALSFRS-r slope, in a 
longitudinal study. Our hypothesis at this step was based on validating the prognostic nature of COL19A1 levels in blood serial samples along the disease progression. For this purpose, both time-dependent Cox regression analysis and linear mixed models were performed in the serial blood cohort. The linear mixed model analysis showed that ALS patients that exhibited high COL19A1 levels from symptom onset also progressed faster during the follow-up period of 24 months from symptom onset (Estimate: -0,0093, CI: $(-0,0181)-(-0,00039) ; P=0,042)$ (Fig. 4A). In addition, time-dependent Cox regression analysis, using leave-one-out cross validation, suggested that the lifespan of sporadic ALS patients showing COL19A1 levels above average (0.819) and a faster progression of the disease (ALSFRS-r slope at sampling scores $\geq 1$ points/month) was reduced $17.9 \%$ (HR: 1.179 , CI: 1.046-1.327, $P=0.007)$, similarly to the result obtained in the blood cohort. Interestingly, ALS patients that showed COL19A1 levels below average from symptom onset (0.819) also showed a longer life expectancy and a light and progressive change in the ALSFRS-r scores at sampling in the follow-up period along disease progression than patients that showed COL19A1 levels above average (Fig. 4B). In accordance with the results obtained in the muscle biopsy and blood cohorts, these findings highlighted the prognostic nature of COL19A1 levels in ALS patients that exhibited both COL19A1 levels above average and ALSFRS-r slope at sampling scores $\geq 1$ points/month in the serial blood cohort, suggesting that COL19A1 levels could be of valuable support in clinical practice when ALSFRS-r slope at sampling was also considered to monitor the ALS patients' group.

The prognostic nature of COL19Al levels in the three cohorts under study is summarized in terms of the average values in COL19A1 levels and the corresponding ALSFRS-r slope at sampling scores (Table 6).

Table 6. Prognostic nature of COL19A1 levels in the three cohorts under study.

\begin{tabular}{|c|c|c|c|c|c|c|}
\hline Group of ALS patients & $\mathbf{N}$ & COL19A1 levels & ALSFRS-r slope & Estimate & HR $(95 \%$ CI $)$ & $P$ value \\
\hline Muscle cohort* & 49 & $\begin{array}{l}\text { above the mean } \\
\text { average }(1.06)\end{array}$ & $\geq 1$ points/month & 0.203 & $1.225(1.058-1.418)$ & 0.007 \\
\hline Blood cohort & 59 & $\begin{array}{l}\text { above the mean } \\
\text { average }(0.819)\end{array}$ & $\geq 1$ points/month & 0.748 & $2.114(1.134-3.941)$ & 0.019 \\
\hline Serial blood cohort & 40 & $\begin{array}{l}\text { above the mean } \\
\text { average }(0.819)\end{array}$ & $\geq 1$ points $/$ month & 0.164 & $1.179(1.046-1.327)$ & 0.007 \\
\hline $\begin{array}{l}\text { Cox regression analysis, using } \\
\text { at first evaluation and ALSFR } \\
\text { lifespan. N: number of enrolle } \\
\text { biopsy cohort, the COL19A1 } \\
\text { COL19A1 gene levels of } 0.81\end{array}$ & & $\begin{array}{l}\text { oss validation, su } \\
\text { apling scores } \geq 1 \\
\text { ALSFRS-r slope } \\
\text { s to the protein le }\end{array}$ & $\begin{array}{l}\text { that in the group o } \\
\text { month in each cor } \\
\text { ling; HR: hazard } \mathrm{r} \\
\text { the Blood cohort } \mathrm{t} \\
5, * ; \mathrm{P}<0.001, * *\end{array}$ & $\begin{array}{l}\text { patients } \\
\text { ading col } \\
\text { CI: confic } \\
\text { dino was }\end{array}$ & $\begin{array}{l}\text { COL19A1 levels above } \\
\text { f patients were associat } \\
\text { interval; } * \text { only in the c } \\
\text { ducible when using the }\end{array}$ & $\begin{array}{l}\text { ean average } \\
\text { th a shorte } \\
\text { the Muscle } \\
\text { ge score for }\end{array}$ \\
\hline
\end{tabular}

\section{DISCUSSION}

This study was designed and performed to identify more reliable prognostic biomarkers for ALS disease progression that could be in close connection with the clinical parameters for a better monitoring and stratification of the patients. The starting point in this study was the skeletal muscle tissue although we also explored blood tissue to identify and validate prognostic biomarkers in a non-invasive way.

In muscle biopsy samples, COL19A1 gene and protein levels were significantly associated with the ALS patient group with respect to healthy controls and patients with ONP, suggesting that COL19A1 levels can be useful as molecular support for diagnosis in this tissue. In the
ALS group, high COL19A1 gene levels were significantly associated with low ALSFRS-r scores, and high COL19A1 protein levels promoted a shorter lifespan in ALS patients that also showed high ALSFRS-r slope scores, and consequently, a faster disease progression. A similar situation was observed in blood samples. COL19A1 and MEF2C levels were associated with the ALS patient group with respect to healthy controls and patients with ONP. In the ALS group, high COL19A1 levels in combination with age at onset, the revised El Escorial criteria, the diagnostic delay and ALSFRS-r scores were associated with a worse prognosis indicating a high disability in the patient. In contrast, high NOGO A and, especially $S N X 10$ levels were related to a better prognosis in ALS patients. However, the prognostic 
nature of these potential biomarkers in blood serial samples were only validated in the case of COL19A1 levels since patients that exhibited high COL19A1 levels and a faster disease progression also showed a shorter lifespan.

Collagen XIX accumulates in the extracellular matrix of restricted tissues, including neural tissue and it seems to modulate cell-matrix interactions and cell-cell communications [14,21]. Recently, the anti-tumor properties of COL19A1 in vitro have also been described, albeit it is involved in the differentiation of muscle cells, central nervous system development, and formation of the esophagus [22]. When muscle fibers are functionally denervated, as it happens in ALS disease, the subsynaptic membrane is able to restore its biochemical and structural organization [23]. The findings observed in muscle biopsy samples suggested that ALS patients that exhibited a faster disease progression they could also exhibited more severe muscle damage. Therefore, high levels of COL19A1 could act as a compensatory response when the disease progression is fast, which is in accordance with previous studies that suggested a close relationship between COL19A1 gene and ALS in muscle biopsies from ALS patients [24]. Furthermore, the remarkable difference in COL19A1 gene and protein levels observed in the ALS patients respect to the ONP group also suggested that COL19A1 could be involved in specific muscle defects that could enhance the desestabilization of motor neuron terminals, contributing especially to the neurodegenerative progression in ALS. Albeit the neuropathies included in this study also share in common with ALS intrinsic muscle defects, neuroinflammation, immune organ dysfunction, metabolic perturbations, defects in neuron excitability and selective motoneuron vulnerability and muscle denervation process, specific processes such as activation of regulatory myogenic factors [14] or the direct cross-talk between NMJ and axonal outgrowth could not be modulated in the same way as in ALS. Structural or functional alterations of skeletal muscle can hinder the transmission of chemical and electrical stimuli from the NMJ to the motor neurons, favoring the dying-back phenomena and therefore contributing to disease pathology [25]. Since COL19A1 is a matrix protein involved in muscle physiology and differentiation [14], alterations in the COL19A1 gene or protein levels could point out tissue damage along disease progression in the neurodegenerative chain of ALS.

In blood samples, high levels of COL19A1 could also represent a repressor stimulus to counteract the denervation processes along disease progression [9,23], and even an overstimulation of immune system in monocytes and dendritic cells by means of leukocyteassociated immunoglobulin-like receptor 1 (LAIR-1) [26]. Recent studies have suggested a novel function of
SNX10 in regulating macrophage polarization and function in mice under inflammatory conditions [27]. Considering that $S N X 10$ has also been reported to be associated with a diminished production of systemic proinflammatory cytokines [27], increasing SNX10 levels could favor macrophage polarization into M2 phenotype, exerting anti-inflammatory action after tissue injury in ALS patients, when the denervation process has started as increasing NOGO A levels suggested, which is in accordance with previous studies [28-32]. Remarkably, it has been reported that thirteen circulating markers of inflammation and of neuromuscular pathology changes were significantly altered in plasma from ALS patients, highlighting the role of inflammatory response and neuromuscular involvement in ALS [33,34]. Moreover, this is an interesting issue since only one recent study has identified blood-derived biomarker from peripheral lymphocytes in ALS patients. In this study, LILRA2, $I T G B 2$ and $C E B P D$ genes were identified as peripherally accessible candidate biomarkers in ALS [35]. These genes are related to the immune system but also they are in close connection with the crosstalk between microglia and motor neuron pathology. The clinical relevance of identifying blood-derived biomarkers from peripheral lymphocytes mainly relies on the fact that it is possible to monitor the impact of other tissues and systems that become damaged during disease progression, such as neuroinflammation, microglial activation or even muscle damage, in parallel with the clinical parameters that can be serially monitored in the patients, at the same time the expression profile of the biomarker candidate is tested in a non-invasive way. In this study, COL19A1 gene improved prognosis accuracy during disease progression not only in patients that were monitored at first evaluation in the blood cohort but also in serial samples and in patients that were monitored longitudinally at 6-monthly intervals during a follow-up period of 24 months, highlighting the prognostic role of this gene in the disease progression and in particular, in the sporadic form of the disease.

The findings provided in this study revealed the potential use of COL19A1 to improve prognosis in the disease progression in a non-invasive way. The combination of high COL19A1 expression levels and a faster disease progression can promote a shorter life expectancy in ALS patients, and therefore COL19A1 levels can be considered a reliable blood-derived biomarker in muscle biopsies and in blood to support the clinical practice and to be of help in future clinical trials, as well as a promising and novel therapeutic target in ALS. 


\section{Acknowledgements}

We especially would like to Dr. Marianne PerreauSaussine from Myobank-AFM of the Institut de Myologie in The Pitié-Salpêtrière Hospital (France) and the Neuromuscular Bank of Tissues and DNA samples, Telethon Network of Genetic Biobank (GTB 12001D to EP) member of the EuroBioBank; the Biobank of Cells, tissues and DNA from patients with neuromuscular diseases, member of the Telethon Network of Genetic Biobanks (Project no. GTB12001), funded by Telethon Italy, and of the EuroBiobank network, which provided us with specimens.

This work was funded by projects PI 14/00088, PI17/00949 and PI17/00491 from the Instituto de Salud Carlos III (Ministry of Economy, Industry and Competitiveness) and cofunded by the European Regional Development Fund, and approved by the Ethics Committee from 12 de Octubre Hospital. The work is supported too by: Consolidated Groups from Gobierno de Aragón; FUNDELA (Spanish Foundation for the Development of ALS Research); Mireia Barneda project "No llores, no te rindas"; "Precipita" crowdfunding campaign thanks to FECyT; and ALS Association (ALSA) \#S54406. The funders had no role in study design, data collection and analysis, decision to publish, or preparation of the manuscript.

\section{References}

[1] Andersen PM, Al-Chalabi A (2011). Clinical genetics of amyotrophic lateral sclerosis: what do we really know? Nat Rev Neurol,7(11):603-615.

[2] Genetics Home Reference. US National Library of Medicine. 29 May 2018. Available from: https://ghr.nlm.nih.gov/condition/amyotrophic-lateralsclerosis.

[3] Jones AR, Woollacott I, Shatunov A, Cooper-Knock J, Buchman V, Sproviero W, et al (2013). Residual association at C9orf72 suggests an alternative amyotrophic lateral sclerosis-causing hexanucleotide repeat. Neurobiol Aging, 34(9):2234.e1-7.

[4] Migliore L, Fontana I, Colognato R, Coppede F, Siciliano G, Murri L (2005). Searching for the role and the most suitable biomarkers of oxidative stress in Alzheimer's disease and in other neurodegenerative diseases. Neurobiol Aging, 26(5):587-595.

[5] Infante J, Prieto C, Sierra M, Sánchez-Juan P, GonzálezAramburu I, Sánchez-Quintana C, et al (2015). Identification of candidate genes for Parkinson's disease through blood transcriptome analysis in LRRK2G2019S carriers, idiopathic cases, and controls. Neurobiol Aging, 36(2):1105-1109.

[6] Rusina R, Ridzon̆ P, Kulišt'ák P, Keller O, Bartos A, Buncová M, et al (2010). Relationship between ALS and the degree of cognitive impairment markers of neurodegeneration and predictors for poor outcome. A prospective study. Eur J Neurol, 17(1):23-30.

[7] Seltman RE, Matthews BR (2012). Frontotemporal lobar degeneration. CNS Drugs, 26(10):841-870.

[8] Kiernan MC, Vucic S, Cheah BC, Turner MR, Eisen A, Hardiman O, et al (2011). Amyotrophic Lateral Sclerosis. Lancet, 377(9769):942-55.

[9] Calvo AC, Manzano R, Atencia-Cibreiro G, Oliván S, Muñoz MJ, Zaragoza P, et al (2012). Genetic biomarkers for ALS disease in transgenic SOD1(G93A) mice. PLoS One, 7(3):e32632.

[10] Gurney ME, Pu H, Chiu AY, Dal Canto MC, Polchow CY, Alexander DD, et al (1994). Motor neuron degeneration in mice that express a human $\mathrm{Cu}, \mathrm{Zn}$ superoxide dismutase mutation. Science, 264(5166):1772-1775.

[11] Raices M, Bukata L, Sakuma S, Borlido J, Hernandez LS, Hart DO, et al (2017). Nuclear Pores Regulate Muscle Development and Maintenance by Assembling a Localized Mef2C Complex. Dev Cell, 41(5):540-554.e7.

[12] Habener A, Chowdhury A, Echtermeyer F, Lichtinghagen R, Theilmeier G, Herzog C (2016). MitoNEET Protects HL-1 Cardiomyocytes from Oxidative Stress Mediated Apoptosis in an In Vitro Model of Hypoxia and Reoxygenation. PLoS One, 11(5):e0156054.

[13] Kobayashi H, Saragai S, Naito A, Ichio K, Kawauchi D, Murakami F (2015). Calm1 signaling pathway is essential for the migration of mouse precerebellar neurons. Development, 142:375-384.

[14] Sumiyoshi H, Mor N, Lee SY, Doty S, Henderson S, Tanaka S, et al (2004). Esophageal muscle physiology and morphogenesis require assembly of a collagen XIXrich basement membrane zone. J Cell Biol, 166:591600.

[15] Stattin EL, Henning P, Klar J, McDermott E, StecksenBlicks C, Sandström PE, et al (2017). SNX10 gene mutation leading to osteopetrosis with dysfunctional osteoclasts. Sci Rep, 7(1):3012.

[16] Finsterer J, Stöllberger C (2013). Apply Awaji-shima consensus conference criteria before diagnosing Amyotrophic Lateral Sclerosis. Open Neurol J, 7:4-6.

[17] Elamin M, Bede P, Montuschi A, Pender N, Chio A, Hardiman O (2015). Predicting prognosis in amyotrophic lateral sclerosis: a simple algorithm. J Neurol, 262(6):1447-1454.

[18] Calvo AC, Moreno-Igoa M, Manzano R, Ordovás L, Yagüe G, Oliván S, et al (2008). Determination of protein and RNA expression levels of common housekeeping genes in a mouse model of neurodegeneration. Proteomics, 8(20):4338-4343.

[19] Florkowski CM (2008). Sensitivity, specificity, Receiver-Operating Characteristic (ROC) Curves and likelihood ratios: communicating the performance of diagnostic tests. Clin Biochem Rev, 29(Suppl 1):S83S87.

[20] Rutkove SB (2015) Clinical Measures of Disease Progression in Amyotrophic Lateral Sclerosis. Neurotherapeutics, 12(2): 384-393. 
[21] Su J., Gorse K, Ramirez F, Fox MA (2010). Collagen XIX is expressed by interneurons and contributes to the formation of hippocampal synapses. J Comp Neurol, 518(2):229-253.

[22] Oudart JB, Monboisse JC, Maquart FX, Brassart B, Brassart-Pasco S, Ramont L (2017). Type XIX collagen: A new partner in the interactions between tumor cells and their microenvironment. Matrix Biol, 57-58: 169177.

[23] Burden SJ, Sargent PB, McMahan UJ (1979). Acetylcholine receptors in regenerating muscle accumulate at original synaptic sites in the absence of the nerve. J Cell Biol, 82(2):412-425.

[24] S htilbans A, Choi SG, Fowkes ME, Khitrov G, Shahbazi M, Ting J, et al (2011). Differential gene expression in patients with amyotrophic lateral sclerosis. Amyotroph Lateral Scler, 12(4):250-256.

[25] Ferraiuolo L, De Bono JP, Heath PR, Holden H, Kasher P, Channon KM, et al. (2009). Transcriptional response of the neuromuscular system to exercise training and potential implications for ALS. J Neurochem, 109(6):1714-24.

[26] Zhou L, Hinerman JM, Blaszczyk M, Miller JL, Conrady DG, Barrow AD, et al (2016). Structural basis for collagen recognition by the immune receptor OSCAR. Blood, 127(5):529-537.

[27] You Y, Zhou C, Li D, Cao ZL, Shen W, Li WZ, et al (2016). Sorting nexin 10 acting as a novel regulator of macrophage polarization mediates inflammatory response in experimental mouse colitis. Sci Reports, 6:20630.

[28] Dupuis L, González de Aguilar JL, di Scala F, Rene F, de Tapia M, Pradat PF, et al (2002). Nogo Provides a
Molecular Marker for Diagnosis of Amyotrophic Lateral Sclerosis. Neurobiol Dis, 10(3):358-365.

[29] Pradat PF, Bruneteau G, González de Aguilar JL, Dupuis L, Jokic N, Salachas F, et al (2007). Nogo-A expression is a prognostic marker in lower motor neuron syndromes. Ann Neurol, 62(1):15-20.

[30] Harel NY, Cudkowicz ME, Brown RH, Strittmatter SM (2009). Serum Nogo-A levels are not elevated in amyotrophic lateral sclerosis patients. Biomarkers, 14(6):414-417.

[31] Yin F, Ye F, Tan L, Liu K, Xuan Z, Zhang J, et al (2012). Alterations of signaling pathways in muscle tissues of patients with amyotrophic lateral sclerosis. Muscle Nerve, 46(6):861-870.

[32] Jokic N, González de Aguilar JL, Pradat PF, Dupuis L, Echaniz-Laguna A, Muller A, et al (2005). Nogo expression in muscle correlates with Amyotrophic Lateral Sclerosis severity. Ann Neurol, 57(4);553-556.

[33] Lu CH, Allen K, Oei F, Leoni E, Kuhle J, Tree T, et al (2016). Systemic inflammatory response and neuromuscular involvement in amyotrophic lateral sclerosis. Neurol Neuroimmunol Neuroinflamm, 3(4):e244.

[34] Lunetta C, Lizio A, Maestri E, Sansone VA, Mora G, Miller RG, et al (2017). Serum C-Reactive Protein as a Prognostic Biomarker in Amyotrophic Lateral Sclerosis. JAMA Neurol, 74(6):660-667.

[35] Cooper-Knock J, Green C, Altschuler G, Wei W, Bury JJ, Heath PR, et al (2017). A data-driven approach links microglia to pathology and prognosis in amyotrophic lateral sclerosis. Acta Neuropathol Comm, 5:23. 